\section{Costo económico de la discapacidad causada por lesiones de tránsito en México durante 2012}

\author{
Economic cost of permanent disability caused by \\ road traffic injuries in Mexico in 2012 \\ Custo econômico da incapacidade causada por \\ acidentes de trânsito no México no ano de 2012
}

\begin{abstract}
This study estimated the economic costs of permanent disability caused by road traffic injuries in Mexico during 2012. From the health system's perspective, a bottom-up approach was used to calculate direct medical costs (hospitalization, outpatient care, rehabilitation, and prostheses). From society's perspective, using a human capital approach, indirect costs were associated with loss of productivity for the victims and their caregivers. Permanent disability due to road traffic injuries takes a high toll on the health system and Mexican society. From the health system perspective, the cost was US\$269,529,480.72, or US\$1,496.33 per victim. The estimated average cost to society was US\$3,445.45 during the first year. The total average cost per victim was US\$4,941.77, resulting in a total economic cost of US\$1,119,761,632.53 during 2012. The study's findings highlight the need to design and implement more rigorous and efficient public polices to control and prevent road traffic injuries in Mexico.
\end{abstract}

Disabled Persons; Accident Consequences; Traffic Accidents; Cost of Illness
Patricia G. Sánchez-Vallejo 1

Ricardo Pérez-Núñez 2

Ileana Heredia-Pi 2

\section{Resumen}

Para estimar el costo económico de la discapacidad permanente causada por lesiones de tránsito en México durante 2012, desde las perspectivas del Sistema de Salud y de la Sociedad, se realizó un estudio de costos que utiliza metodología bottom-up, considerando costos directos médicos (hospitalización, consultas ambulatorias y de rehabilitación y prótesis), y costos indirectos (pérdida de productividad del lesionado y cuidador) con una aproximación de capital humano. La discapacidad causada por lesiones de tránsito tiene un alto costo para el sistema de salud y la sociedad mexicana. Desde la perspectiva del sistema de salud, el costo en pesos mexicanos de la discapacidad permanente fue de US\$269.529.480,72, equivalente a US\$1.496,33 por persona. Desde la perspectiva de la sociedad, se estimaron US\$3.445,45 durante el primer año. En promedio, se estimó un costo total de US\$4.941,77 por persona, resultando en un total de US\$1.119.761.632,53 en 2012. Los resultados de este estudio evidencian la necesidad de disenar e implementar políticas más enérgicas y eficientes para el control de las lesiones de tránsito en México.

Personas com Discapacidad; Consecuencias de Accidentes; Accidentes de Tránsito; Costo de Enfermedad 


\section{Introducción}

Las lesiones causadas por el tránsito son la principal causa de muerte por lesiones en los países de ingresos bajos y medios 1. En países de ingresos medios, las lesiones causadas por el tránsito ocupaban el sexto lugar en 2004 en la lista de las diez causas principales de mortalidad 2 , y, además, representaban el $22 \%$ de los años de vida perdidos (AVP) por muertes prematuras asociadas a lesiones 3. En México, las lesiones causadas por el tránsito son la primera causa de muerte por lesiones no intencionales ${ }^{4}$, en donde además aproximadamente ocho de diez fallecidos son hombres 5 .

Sin embargo, es importante destacar que no todas las lesiones son fatales. Se ha estimado que cada habitante del mundo, durante toda su vida, tiene la probabilidad de uno en siete de sufrir, algún día, una lesión causada por el tránsito ${ }^{6}$. Según la Encuesta Nacional de Salud y Nutrición de 2012 (ENSANut 2012), 1,4 millones de personas sufrieron lesiones de tránsito no fatales 7 . Esto implica que por cada fallecido hay entre $35^{8} \mathrm{y}$ 679 lesionados aproximadamente. Así, en países de ingresos bajos y medios, las lesiones causadas por el tránsito generan entre 30 y $86 \%$ del total de admisiones por lesiones en los establecimientos de salud 10. Por otra parte, dado que las lesiones causadas por el tránsito afectan principalmente a la población en edad productiva, los costos económicos en términos de tratamiento médico, rehabilitación y pérdida de productividad tienden a ser altos con repercusiones económicas importantes para las sociedades 11 .

De las personas lesionadas, un porcentaje relativamente alto se recupera, pero otras nunca lo hacen completamente y sufren algún tipo de discapacidad. La Organización Mundial de la Salud (OMS) estimó recientemente que alrededor de $15,6 \%$ de las personas de 18 y más años viven con algún tipo de discapacidad, lo que varió de $11,8 \%$ en países de altos ingresos a $18 \%$ en países de bajos ingresos. Entre 2,2\% y 3,8\% esta discapacidad es considerada severa 12 . En México, el Censo de Población y Vivienda del 2000 evidenció que un 1,8\% de la población total, tenía algún tipo de discapacidad 13. Según la Encuesta $\mathrm{Na}$ cional de Salud de 2000, la proporción es mayor $(2,3 \%)$ y en $20,1 \%$ de los casos la discapacidad fue causada por lesiones no intencionales 14 . Los AVP derivados de la discapacidad constituyen aproximadamente $18 \%$ en México, por encima de la media global de $14^{3}$. La discapacidad causada por lesiones es un evento que trae consigo un alto costo para la salud pública en términos de pérdidas monetarias y sufrimiento personal 15 .
La discapacidad ha sido abordada desde dos perspectivas diferentes: la médica y la social. El modelo médico entiende la discapacidad como una característica inherente a la persona, originada por alguna enfermedad, trauma o condición médica. De ahí que requiera atención y tratamiento médico para "corregir" el problema a nivel individual. Por otro lado, el modelo social ve en la discapacidad un problema social creado por las actitudes y características del ambiente social, por lo que la discapacidad requiere una respuesta política. Dado que ningún modelo por sí solo es adecuado, y ambos son parcialmente válidos, la OMS lideró la propuesta de un modelo biopsicosocial que pretende integrar ambos modelos 16 .

De acuerdo con esta propuesta, la discapacidad y el funcionamiento son vistos como resultados de la interacción entre condiciones de salud (enfermedades, desórdenes y lesiones) y factores del contexto como los ambientales (actitudes sociales, estructura legal y social, clima, características arquitectónicas, etc.) y los personales (edad, género, escolaridad, antecedentes sociales, profesión, experiencias pasadas y presentes, entre otras). Desde este enfoque, se identifican tres niveles de funcionamiento humano: a nivel corporal o de una parte del cuerpo; a nivel de la persona y a nivel de toda persona en un contexto social. La discapacidad, por tanto, implica la disfunción en una o más de los mismos niveles: problemas en la función corporal, limitación de las actividades cotidianas y restricción de la participación social 16. Así, la discapacidad causada por el tránsito no debería ser entendida solamente como un estado físico o emocional, sino como fenómeno socioeconómico con un proceso dinámico que fluctúa en extensión y severidad a lo largo de la vida, y puede limitar o no la capacidad para trabajar y vivir de forma independiente 17 .

En México, no existen estudios previos que documenten específicamente el costo que implica para el país la discapacidad causada por lesiones de tránsito. Dado que las lesiones causadas por el tránsito, y por tanto sus consecuencias negativas, son prevenibles, esta información contribuiría a dimensionar la magnitud real de este problema de salud pública al conocer el impacto que tienen sobre la sociedad y el sistema de salud. La evidencia disponible ha mostrado cómo las lesiones causadas por el tránsito y su costo económico afectan de forma diferenciada a los distintos usuarios de la vía pública, siendo mayores sus consecuencias en los usuarios más vulnerables 18,19 . De ahí que este estudio estima el costo económico de la discapacidad permanente causada por lesiones de tránsito en México durante 2012, por tipo de actor vial y desde la 
perspectiva del Sistema de Salud como pagador. Adicionalmente, se estimó el costo indirecto que representa para la sociedad la pérdida de productividad, asociada a la discapacidad permanente, ampliando así la perspectiva del estudio.

\section{Metodología}

\section{Diseño y tipo de estudio}

Se realizó un estudio de costos que, primeramente, utilizó información proveniente de la ENSANut - 2012 20. Se trata de una encuesta de naturaleza probabilística y, por su diseño, representativa del nivel nacional, estatal y por estratos urbano/rural. Más información sobre su diseño ha sido publicada previamente 21 . En particular, se analizó información de las secciones de “accidentes" y "discapacidad", pertenecientes a los cuestionarios dirigidos a niños, adolescentes y adultos. Esta información permitió estimar la prevalencia de las lesiones causadas por el tránsito y la discapacidad asociada con dichas lesiones. A partir de estas estimaciones, utilizando la metodología bottom-up, desde las perspectivas de la sociedad y del sistema de salud, se estimó el costo económico de la discapacidad permanente causada por lesiones de tránsito en México, durante 2012. La metodología bottom-up utiliza los costos unitarios de los servicios y recursos utilizados en la atención médica y aplica estos datos al número total de eventos relacionados con la enfermedad, observados para cada paciente de la muestra analizada, para llegar a una estimación de los costos de atención de la enfermedad y a estimaciones de los costos promedios de los servicios por paciente 22,23 .

El análisis consideró los costos directos médicos (hospitalización, consultas ambulatorias posteriores al evento de hospitalización, consultas de rehabilitación y la utilización de prótesis). Adicionalmente, se estimaron los costos indirectos asociados con la pérdida de productividad de las personas con discapacidad permanente, derivada de lesiones de tránsito y de sus cuidadores, utilizando la aproximación metodológica del capital humano, para la cual se fijó la edad de jubilación en 65 años, se consideró el salario mínimo para México en 2012 (escenario basal) y una esperanza de vida de 74 años. En el primer trimestre del 2012, aproximadamente, $13,52 \%$ de la población ocupada -aproximadamente 5,6\% de la población total- recibía solamente un salario mínimo, de acuerdo con datos de la Encuesta Nacional de Ocupación y Empleo. Es importante mencionar que en el primer trimestre del 2012, de la población total, aproximadamente 41,16\% se encontraba económicamente activa 24 . Por medio del método del Capital Humano, se estima la pérdida de productividad por morbi-mortalidad en términos de pérdidas de salario o ingresos del paciente o los familiares a su cuidado, a partir de la valoración de la disminución de las horas de trabajo y/o del nivel de producción, como consecuencia de la enfermedad 22,23.

Los precios fueron ajustados a los valores del 2012, utilizando el índice de precios al consumidor cuando fue necesario 25 . Todos los costos fueron convertidos a dólares de los Estados Unidos para facilitar su comparabilidad internacional. En promedio durante 2012 un peso mexicano equivalía a 0,077 dólares de los Estados Unidos (http://www.banxico.org.mx/portal-mercadocambiario/index.html/).

\section{- Estimación del número de personas con discapacidad permanente}

En virtud de la falta de información apropiada, la definición operacional de discapacidad utilizada en este estudio responde más bien al modelo médico. El cálculo del número de personas con discapacidad permanente por lesiones de tránsito se realizó con base en el total de lesionados de tránsito identificados a partir de la ENSANut 2012 20. La encuesta estimó para dicho año 1.377.625 lesionados por tránsito, que representa el 1,19\% de la población total del país. A ellos se les preguntó si sus lesiones les habían ocasionado un problema de salud permanente. Un total de 226.591 personas (IC95\%: 176.198-287.924) auto-reportaron discapacidad permanente, cifra que representa $16,45 \%$ del total de lesionados en el tránsito 7. El valor puntual de esta variable fue utilizado en la estimación de la población de lesionados con discapacidad permanente, considerada en el escenario basal.

\section{- Estimación de los costos directos por utilización de insumos y servicios de salud en personas con discapacidad permanente, derivada de lesiones de tránsito}

Los costos de hospitalización, de consultas ambulatorias y de consultas de rehabilitación, durante 2012 se estimaron utilizando información financiera procedente de 3 fuentes de información: (i) del Hospital General Dr. Manuel Gea González (HGDMGG) 26, (ii) la base de datos WHO-CHOICE 27, y (iii) el Instituto Mexicano del Seguro Social (IMSS) 28 , lo que permitió definir, respectivamente, 3 escenarios distintos para las estimaciones de costos: un escenario "optimista” (costos más bajos), un escenario "basal” (costos 
medios) y un tercer escenario "pesimista" (costos más altos).

Se asumió que el $100 \%$ de los lesionados por el tránsito que terminan con una discapacidad permanente, utilizaron servicios hospitalarios por el evento que originó su discapacidad. Aunque se sabe que en México existen personas con discapacidad permanente que no son hospitalizadas, el porcentaje exacto no ha sido documentado previamente, por lo que no fue posible considerarlo en esta estimación. Los días de estancia hospitalaria para cada paciente se calcularon a partir del estudio Las Lesiones por Causa Externa en México 10,29. La información respecto al número de consultas ambulatorias y de rehabilitación utilizadas durante un año por cada persona con discapacidad permanente, derivada de lesiones de tránsito (intensidad de utilización aproximada de dichos servicios) se obtuvo de lo observado en estudios previos 9,11.

Los costos derivados de la hospitalización se construyeron multiplicando el número de ingresos hospitalarios, la estancia hospitalaria promedio tomada del estudio de Ávila-Burgos et al. ${ }^{29}$ y el costo día-cama. De igual forma, el costo asociado a consultas ambulatorias y consultas de rehabilitación se calculó multiplicando el número calculado de pacientes que utilizan dichos servicios, por el costo de los mismos. Finalmente, para estimar el costo de la utilización de aparatos de prótesis, se multiplicó el costo de las prótesis por el número de pacientes que necesitaron de este insumo estimado, utilizando información documentada en trabajos previos realizados en el país 9. Para la estimación de los costos directos futuros (costos por utilización de servicios de salud durante toda la esperanza de vida del lesionado con discapacidad permanente) se utilizó información sobre el gasto en salud promedio por discapacitado, estimado a partir de la Encuesta Nacional sobre la Percepción de la Discapacidad en la Población Mexicana 201030. A partir de esta encuesta, se consideró un costo anual promedio de US\$781,86 durante los primeros 10 años, consecutivos al momento de la lesión y un costo promedio anual de US\$205,44 para los años subsiguientes. Estos costos fueron descontados utilizando tasas de descuento del $5 \%$ y del $10 \%$. Los parámetros utilizados para la estimación de los costos directos e indirectos se presentan en la Tabla 1.

Así, para la estimación del costo directo total se utilizó la siguiente fórmula:

$$
\begin{aligned}
& C D_{j}=\sum_{i=1}^{n} C I H_{i j}+\sum_{i=1}^{n} C C A_{i j}+\sum_{i=1}^{n} C C R_{i j}+\sum_{i=1}^{n} C P_{i j} \\
& \text { Donde: } \\
& C D_{j}=\text { Costo directo del usuario vial } j ;
\end{aligned}
$$

$\mathrm{CIH}_{j}=$ Costo de ingresos hospitalarios;

$C C A_{j}=$ Costo de consultas ambulatorias;

$C C R_{j}=$ Costo de consultas de rehabilitación;

$C P_{j}=$ Costo de prótesis;

$i=$ número del individuo $(\mathrm{i}=1,2,3, \ldots, \mathrm{n})$.

\section{Estimación de los costos indirectos}

Se calculó la pérdida de productividad, según usuario vial, usando la aproximación metodológica del capital humano. En una primera estimación se calcularon los costos indirectos sólo para el primer año transcurrido, a partir del momento de la ocurrencia de la lesión que derivó en una discapacidad permanente. Adicionalmente, con la intención de ofrecer una estimación a largo plazo, en una segunda estimación, se calcularon los costos indirectos proyectados a futuro por tipo de lesionado, para los años subsecuentes a la lesión, considerando la esperanza de vida para los lesionados con discapacidad 31,32 y tomando como referencia información de la Encuesta Nacional sobre la Percepción de la Discapacidad en la Población Mexicana 2010 30. Estos costos también fueron descontados utilizando tasas de descuento del 5\% y del 10\%. Para estos cálculos se consideró la edad promedio de cada usuario vial, en el momento de sufrir la lesión causante de la discapacidad permanente, según la información disponible de la ENSANut 2012. A partir de esta información, se calcularon los años de vida productiva perdidos. De esta forma, la pérdida de productividad laboral a largo plazo se calculó multiplicando los años productivos perdidos (se fijó la edad de jubilación en 65 años), por el salario anual calculado a partir del salario mínimo vigente en el Distrito Federal en 2012 33, para los escenarios optimista y basal. Para estimar el costo indirecto asociado a la pérdida productiva en el escenario pesimista, se utilizó el ingreso promedio reportado en la Encuesta Nacional de Ingresos y Gastos en los Hogares 24 en vez de salario mínimo, con el fin de aproximar un costo más realista para ese escenario. La información sobre el tiempo que un cuidador destina a la persona con discapacidad, se obtuvo del estudio $L a$ Atención de los Enfermos y Discapacitados en el Hogar. Retos para el Sistema de Salud Mexicano de Nigenda et al. 32 .

De esta forma, los costos indirectos para cada individuo con discapacidad permanente se estimaron utilizando la siguiente fórmula:

$$
\begin{aligned}
& C I_{j}=\sum_{i=1}^{n}\left[\left(D E H_{i j}+D C A_{i j}+D R C_{i j}\right)\left(I_{i j}\right)\right] \\
& +\sum_{i=1}^{n}\left(D C_{i j} * I_{i j}\right)+\sum_{i=1}^{n}\left(P P_{i j}\right)
\end{aligned}
$$


Tabla 1

Parámetros utilizados en las estimaciones de costos directos e indirectos.

\begin{tabular}{|c|c|c|c|}
\hline \multirow[t]{2}{*}{ Servicios } & \multicolumn{3}{|c|}{ Tiempo de utilización de los servicios } \\
\hline & \multicolumn{2}{|c|}{ Promedio de uso } & \multirow{2}{*}{$\begin{array}{c}\text { Referencia } \\
10,29\end{array}$} \\
\hline Hospitalización (días) & 5,4 & & \\
\hline & \multicolumn{3}{|c|}{ Utilización de los servicios } \\
\hline & $\begin{array}{l}\text { Promedio de uso } \\
\text { por año }\end{array}$ & $\begin{array}{l}\text { Porcentaje de personas } \\
\text { con discapacidad } \\
\text { permanente derivada de } \\
\text { lesiones de tránsito que } \\
\text { utilizan servicio }\end{array}$ & Referencia \\
\hline Consultas ambulatorias (número promedio anual) & 21 & $77,24 \%$ & 9,11 \\
\hline Consultas de rehabilitación (número promedio anual) & 19,37 & $81,8 \%$ & 9,11 \\
\hline Prótesis & ND & $63,29 \%$ & 9 \\
\hline
\end{tabular}

\begin{tabular}{lccc}
\hline & Costo de los servicios utilizados & Costo (USD) & Referencia \\
\hline Día-paciente de hospitalización & Pesimista & 391,00 & 108,50 \\
& Basal & 104,53 & 28 \\
Consultas ambulatorias & Pesimista & 37,11 & 27 \\
& Basal & 19,01 & 26 \\
Consultas de rehabilitación & Optimista & 6,00 & 27 \\
& Pesimista & 60,75 & 26 \\
Prótesis & Basal & 19,01 & 28 \\
Gasto en salud durante los primeros 10 años & Optimista & 2,93 & 26 \\
Gasto en salud después de 10 años & & 99,43 & 9 \\
\end{tabular}

Parámetros utilizados en las estimaciones de los costos indirectos

Promedio de uso

Referencia
Estancia hospitalaria (días)

Consultas ambulatorias (número promedio anual)

Tiempo de recuperación en casa (días)

Tiempo del cuidador para personas con discapacidad permanente derivada de lesiones de tránsito (días)

Salario mínimo (diario, USD)

Ingreso promedio (diario, USD)

Edad promedio en el momento de la lesión causante de la

discapacidad permanente (años)
ND: no disponible.

Fuente: elaboración propia a partir de la bibliografía consultada. Las referencias específicas para cada parámetro se encuentran en la columna de extrema derecha.

$\begin{array}{cc}5,4 & 29 \\ 21 & 9 \\ 303,24 & 9 \\ 124,25 & 32\end{array}$

4,80

$6,73-24$

$35,28-20$


Donde:

$C i_{j}=$ costo indirecto del usuario vial $j$; $D E H_{j}=$ promedio de días de estancia hospitalaria;

$D C A_{j}=$ promedio de días de consultas ambulatorias;

$D R C_{j}=$ promedio de días de recuperación en casa;

$I_{j}=$ salario o ingreso diario perdido;

$D C_{j}=$ promedio de días que un cuidador dedica a la persona con discapacidad;

$P P_{j}=$ pérdida de productividad (salario anual perdido por discapacidad permanente).

Finalmente, los costos totales individuales de la discapacidad permanente, causada por el tránsito para este estudio, se obtuvieron sumando los costos directos y los costos indirectos previamente estimados.

\section{Análisis de la información}

Se realizó un análisis descriptivo para todas las variables estudiadas. El análisis de los datos se realizó con ayuda de los programas Stata 12.1 (Stata Corp., College Station, EEUU) y Microsoft Excel versión 2010 (Microsoft Corp., EEUU). La Figura 1 muestra el modelo analítico utilizado para la sistematización de la información obtenida, y la secuencia en la que se manejaron los datos para la estimación de los costos directos. Los resultados se presentan por tipo de usuario vial. Para fines de este estudio, los pasajeros de vehículos de motor de cuatro o más ruedas fueron considerados como "automovilistas".
Debido a la incertidumbre en algunos de los parámetros utilizados, se realizó un análisis de sensibilidad determinístico, utilizando los tres escenarios alternativos de costos institucionales (optimista, basal y pesimista) detallados previamente. Adicionalmente, se consideró la incertidumbre en el número de lesionados con discapacidad, estimado a partir de la ENSANut 2012, para el cual se utilizó el valor puntual de esta variable en el escenario basal, mientras el límite inferior de su intervalo de confianza al 95\% (IC95\%) fue considerado para el escenario optimista y el límite superior para el escenario pesimista. Finalmente, en la estimación de los costos indirectos, por pérdida de productividad del lesionado con discapacidad y su cuidador, se consideró el salario mínimo para los escenarios optimista y basal y el salario promedio para el escenario pesimista.

\section{Resultados}

\section{Costos directos asociados a la discapacidad}

El costo de hospitalización por persona con discapacidad al año fue de US\$585,90, en nuestro escenario basal (Tabla 2). El costo aproximado asociado a la utilización de las consultas ambulatorias fue de US\$399,40 por lesionado, asumiendo que el porcentaje de utilización de este servicio fue de $77,24 \%$ y que la frecuencia de uso fue de 21 consultas por año. En el caso de las consultas o sesiones de rehabilitación, se asumió el

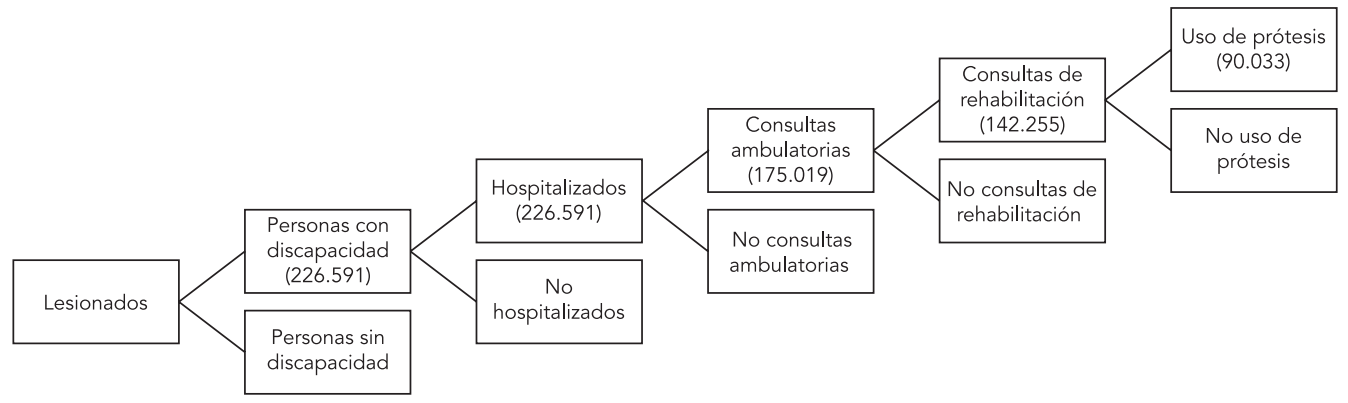

Fuente: elaboración propia a partir de los datos epidemiológicos obtenidos de la Encuesta Nacional de Salud y Nutrición de 201220 y de Pérez-Núñez et al. ${ }^{9}$. 
Costos directos médicos de la discapacidad permanente por lesiones causadas por el tránsito. México, 2012.

\begin{tabular}{|c|c|c|c|}
\hline Concepto & $\mathrm{n}$ & Costo por persona (USD) & Costo total (USD) \\
\hline Ingresos hospitalarios & 226.591 & 585,90 & $132.760 .523,41$ \\
\hline Peatón & 40.130 & & $23.512 .318,69$ \\
\hline Ciclista & 16.609 & & $9.731 .275,88$ \\
\hline Motociclista & 59.122 & & $34.639 .803,28$ \\
\hline Automovilista & 110.730 & & $64.877 .125,56$ \\
\hline Consultas ambulatorias & 175.019 & 399,40 & $69.902 .369,01$ \\
\hline Peatón & 30.996 & & $12.379 .935,96$ \\
\hline Ciclista & 12.829 & & $5.123 .806,54$ \\
\hline Motociclista & 45.666 & & $18.238 .887,95$ \\
\hline Automovilista & 85.528 & & $34.159 .738,56$ \\
\hline Sesiones de rehabilitación & 142.255 & 399,40 & $56.816 .645,53$ \\
\hline Peatón & 25.194 & & $10.062 .411,95$ \\
\hline Ciclista & 10.427 & & $4.164 .629,95$ \\
\hline Motociclista & 37.117 & & $14.824 .568,13$ \\
\hline Automovilista & 69.517 & & $27.765 .035,50$ \\
\hline Uso de prótesis & 90.033 & 111,62 & $10.049 .942,77$ \\
\hline Peatón & 15.945 & & $1.779 .877,41$ \\
\hline Ciclista & 6.599 & & $736.655,47$ \\
\hline Motociclista & 23.491 & & $2.622 .225,58$ \\
\hline Automovilista & 43.997 & & $4.911 .184,30$ \\
\hline
\end{tabular}

Fuente: elaboración propia a partir de los datos epidemiológicos obtenidos de la Encuesta Nacional de Salud y Nutrición de 201220 y de Pérez-Núñez et al. 9.

mismo costo que el de las consultas ambulatorias, con un porcentaje de utilización de $81,28 \%$ y una frecuencia de uso de $19,37 \%$ consultas al año por lesionado. Para el último componente, el uso de prótesis, el costo resultante fue de US\$111,62 por su utilización or lo tanto desde la perspectiva del sistema de salud, el costo de la discapacidad permanente en 2012 fue de US\$269.529.480,72, lo que equivale a US $\$ 1.496,33$ por persona. Los costos hospitalarios representan el rubro de mayor peso relativo entre todos los costos directos considerados por persona $(39,16 \%)$. Adicionalmente, la Tabla 3 presenta los costos económicos a futuro, estimados para cada uno de los usuarios de la vía pública. Se puede observar que el costo total a futuro es mayor en motociclistas y menor en peatones.

\section{Costos indirectos asociados a la discapacidad por lesiones de tránsito para 2012}

De acuerdo a nuestras estimaciones, los costos indirectos asociados a la pérdida de productividad de la persona con discapacidad, tomando en cuenta los días e ingresos perdidos por estancia hospitalaria, por asistir a las consultas ambulatorias y durante la recuperación en casa, ascienden a US\$1.582,08 por persona al año. Las estimaciones de la pérdida productiva del individuo con discapacidad arrojaron un resultado de US\$1.267,04. Finalmente, respecto a la pérdida de productividad de los cuidadores, considerando los días de productividad y el ingreso perdidos por el cuidador, relacionados con el cuidado de la persona con discapacidad muestran un costo aproximado de US\$596,32. Así, el costo indirecto durante el primer año se estimó en US\$3.445,45. La distribución de los costos totales indirectos por grupo de usuario vial se muestra en la Tabla 4 . En ella también se expone que los automovilistas fueron los usuarios con la mayor pérdida de productividad, con un aproximado de US\$381.514.506,30 durante 2012.

\section{Costos totales de la discapacidad permanente por lesiones de tránsito en 2012}

En promedio se estimó un costo total de US\$4.941,77 por usuario vial, lo que significa que aproximadamente la discapacidad permanente costó US\$1.119.761.632,53 en 2012. En la Tabla 5 
Tabla 3

Costos a futuro relacionados con la discapacidad permanente por lesiones causadas por el tránsito, según tipo de usuario vial, descontado al $5 \%$ y al 10\% (costos presentados en dólares americanos).

\begin{tabular}{lccc}
\hline Usuario vial & Costo total (USD) & Descuento al 10\% (USD) & Descuento al 5\% (USD) \\
\hline Peatón & $11.311,25$ & $1.131,12$ & $2.262,25$ \\
Ciclista & $13.981,88$ & $1.398,19$ & $2.796,38$ \\
Motociclista & $14.392,74$ & $1.439,27$ & $2.878,55$ \\
Automovilista & $13.776,44$ & $1.377,64$ & $2.755,29$ \\
\hline
\end{tabular}

Fuente: elaboración própria.

Tabla 4

Costo indirecto total por grupo de usuario vial en México, descontada al $5 \%$ y al $10 \%$ (costos presentados en dólares americanos)

\begin{tabular}{lccc}
\hline Tipo de usuario & Costo indirecto total (USD) & Descuento al 10\% (USD) & Descuento al 5\% (USD) \\
\hline Peatón & $138.127 .718,38$ & $12.569 .622,37$ & $23.044 .307,68$ \\
Ciclista & $57.168 .284,94$ & $5.202 .313,93$ & $9.537 .575,54$ \\
Motociclista & $203.498 .304,65$ & $18.518 .345,72$ & $33.950 .300,49$ \\
Automovilista & $381.133 .372,93$ & $34.683 .136,94$ & $63.585 .751,05$ \\
\hline
\end{tabular}

Fuente: elaboración própria.

Tabla 5

Comparación de costos para escenarios alternos, 2012 (costos presentados en dólares americanos).

\begin{tabular}{lccc}
\hline & & Escenarios \\
& Optimista (USD) & Base (USD) & Pesimista (USD) \\
\hline Costos directos (subtotal) & 858,88 & $1.496,33$ & $4.179,13$ \\
Hospitalización & 564,45 & 585,90 & $2.111,43$ \\
Consultas ambulatorias & 126,13 & 399,40 & 779,39 \\
Consultas rehabilitación & 56,68 & 399,40 & $1.176,79$ \\
Prótesis & 111,62 & 111,62 & 111,51 \\
Costos indirectos (subtotal) & $3.445,45$ & $3.445,45$ & $4.827,96$ \\
Costos total & $4.304,32$ & $4.941,77$ & $9.007,08$ \\
\hline
\end{tabular}

Fuente: elaboración própria.

se muestran los resultados del análisis de sensibilidad. El rubro de los costos directos fue el que mayores diferencias mostró, el escenario pesimista fue poco más de cuatro veces más caro que el optimista, mientras que los costos indirectos del escenario pesimista fueron poco más de $40 \%$ más altos que el optimista. Comparando los costos totales por persona con discapacidad perma- nente, derivada de lesiones de tránsito, notamos que el escenario optimista es un $13 \%$ menor que el escenario basal, mientras que el escenario pesimista es casi un $82 \%$ mayor que el costo total del escenario basal. 


\section{Discusión}

El presente estudio representa un primer esfuerzo por dimensionar las consecuencias económicas, para el sistema de salud y la sociedad, de la discapacidad permanente causada por lesiones de tránsito en México. Esta información contribuye directamente a ampliar el panorama de conocimiento respecto al estudio de las lesiones de tránsito en México y sus consecuencias no fatales. Al darle un valor económico a la carga que la discapacidad secundaria a lesiones causadas por el tránsito representa para la sociedad y el sistema de salud, se permite dimensionar la magnitud de este problema de salud pública $y$, con esto, justificar la urgente necesidad de implementar programas y políticas públicas que permitan evitar estas consecuencias negativas para la salud de la población. Los recursos que la sociedad mexicana pierde anualmente como consecuencia de este problema de salud pública podrían ser destinados para atender otros problemas prioritarios del sector salud o de la sociedad en su conjunto. Así, por ejemplo, a partir de nuestras estimaciones, se puede observar cómo la sociedad mexicana podría destinar un total de 411.074 becas anuales a familias en extrema pobreza (tomando como referencia el monto máximo mensual del programa Oportunidades que es alrededor de US\$212,91 al mes, según registros de Secretaría de Desarrollo Social 34, en lugar de perderlo anualmente por esta causa.

Hasta nuestro conocimiento, no existen estudios en Latinoamérica que hayan documentado el costo de la discapacidad secundaria a lesiones causadas por el tránsito, lo que dificulta comparar nuestros resultados. En algunos países de altos ingresos se ha cuantificado la magnitud de la discapacidad por lesiones de tránsito en términos de costos económicos, aunque vale la pena reconocer que no son necesariamente comparables con México. Utilizando el método del capital humano, en 1997 Albert \& Cloutier 35 reportaron un costo indirecto de 104 millones de dólares por discapacidad en la provincia de Alberta en Canadá. Posteriormente en 2004, Bastida et al. 36 realizaron una estimación de los costos económicos de las lesiones causadas por el tránsito en España, y encontraron que la pérdida de productividad por retiro temprano, ascendía a $€ 1.597,78$ millones (US\$1.910.124.228,91). Respecto a los costos indirectos, nuestras estimaciones muestran un valor monetario diez veces mayor al reportado por Albert \& Cloutier 35, mientras que el costo reportado por Bastida et al. 36 duplica el costo documentado en nuestro estudio. Estas diferencias pueden deberse al tamaño de las poblaciones principalmente, y en un segundo lugar, a los métodos empleados, por ejemplo Bastida et al. 36, incluyeron las muertes prematuras en su estimación de costos indirectos.

Una de las principales barreras a las que nos enfrentamos en la construcción de este estudio, es la limitación de información respecto a la utilización de servicios de salud por parte de personas con discapacidad en general, y mucho más limitada es la información desagregada por origen de la discapacidad. Además, debemos señalar que posiblemente existe una subestimación de los costos directos totales, pues no se tomaron en cuenta los costos directos no médicos, como los costos de traslado y trasporte durante la búsqueda de atención a la salud, así como el costo de las adaptaciones a la vivienda y a los vehículos de transporte (cuando las hubo). Adicionalmente, se utilizaron algunos parámetros que fueron tomados de fuentes internacionales, con el consiguiente riesgo de que su magnitud no refleje con exactitud la realidad en México.

Por otro lado, la estimación del número de discapacitados permanentes podría estar sobreestimada por una posible tendencia a auto-reportar más esta condición. En China 37 y Tailandia ${ }^{38}$ se ha reportado que el $4,8 \%$ y el $4,6 \%$ de las personas que presentan lesiones causadas por el tránsito sufren discapacidad permanente, lo que es mayor a lo observado en este estudio (16,45\%). Otros estudios han documentado un mayor porcentaje. En Nigeria, Juillard et al. 39 reportaron que $29,1 \%$ de personas lesionadas en el tránsito tuvieron discapacidad permanente y en $67,6 \%$ de ellos la discapacidad limita la realización de sus actividades de la vida diaria. Nuestro estudio utiliza la mejor fuente de información disponible por el momento para estimar la incidencia anual de discapacidad por esta causa. Se hace evidente, sin embargo, la necesidad de construir mecanismos para cuantificar la discapacidad por lesiones de tránsito, y cualquier otro tipo de discapacidad, de una manera más exhaustiva, incluyente y crítica. Así, futuros estudios deberán transitar hacia una conceptualización más integral de la discapacidad que, por una parte, incluya no sólo el auto-reporte de esta condición por las propias personas (necesidad sentida), sino también criterios técnicos que permitan delimitar lo que debería ser considerado como discapacidad; y que por la otra parte integre la dimensión médicobiológica con la social como ha sido sugerido por la OMS 16.

No obstante, entre las fortalezas de nuestro análisis destaca el hecho de que se realizaron estimaciones para tres escenarios diferentes que permiten dimensionar dentro de un intervalo de incertidumbre amplio la magnitud del problema y reducir la incertidumbre asociada a los pará- 
metros utilizados. Esta información aporta al establecimiento de prioridades y prácticas acorde con el Reporte de Seguridad Vial de la OMS de 2013 y con el Reporte Mundial sobre Discapacidad también de la OMS. Por ejemplo, es necesario crear métodos de recolección de datos uniformes, de forma sistemática, para documentar de una mejor manera el número de personas con discapacidad por lesiones de tránsito, información útil para la toma de decisiones. Esto permitirá guiar los servicios de planeación del país 40 , y diseñar programas, intervenciones y políticas adecuadas para la prevención y atención de la discapacidad 12 .

A pesar de los avances respecto al estudio sobre las lesiones causadas por el tránsito, y sobre las políticas formuladas para evitarlas, los resultados de este estudio evidencian la necesidad de diseñar e implementar políticas más enérgicas y eficientes para su prevención y control en el país. Para ello se debe prestar especial atención a los usuarios de la vía pública más vulnerables (peatones, ciclistas y motociclistas), pues en conjunto son los mayormente afectados y a los que tradicionalmente menos atención se les presta. De igual manera, sería importante que futuros estudios aborden el tema de la discapacidad desde diferentes perspectivas, como por ejemplo, desde el punto de vista de la calidad técnica de la atención médica recibida, principalmente en la atención prehospitalaria en donde algunos au- tores han reportado distintos problemas de coordinación y regulación ${ }^{41}$ o falta de capacitación del personal que provee estos servicios 42 . Otras perspectivas incluyen, la accesibilidad a los servicios de salud (desde atención prehospitalaria, hospitalaria y de rehabilitación) o también desde el punto de vista del gasto catastrófico y el empobrecimiento de las familias derivados de los altos costos de atención y de las pérdidas productivas de lesionados y familiares, entre otras.

Nuestro estudio constituye un primer esfuerzo para medir la carga económica de la discapacidad secundaria a eventos de tránsito en México que busca, además, incentivar futuros estudios que puedan cubrir las brechas metodológicas y de información aquí documentadas. Conocer el costo económico de la discapacidad, permite dimensionar de mejor forma un problema que parece no reconocerse en la sociedad mexicana. Así, esta información constituye una herramienta de suma importancia para planear y diseñar estrategias para la atención de la discapacidad además de que sustenta las bases para futuras evaluaciones económicas integrales de intervenciones y programas destinados a prevenir o controlar este problema de salud pública. Además permite planear de manera adecuada la distribución de los recursos, y en este caso en particular, permite contrastar el costo de la incidencia actual de discapacidad causada por lesiones de tránsito contra el costo de prevenirla.

\section{Resumo}

Para estimar o custo econômico da incapacidade permanente causada por acidentes de trânsito no México no ano de 2012, com base nas perspectivas do Sistema de Saúde e da sociedade, foi realizado um estudo de custos utilizando-se a metodologia bottom-up, considerando por um lado os custos diretos médicos (hospitalização, consultas ambulatoriais e de reabilitação e próteses) e, por outro, os custos indiretos associados à perda de produtividade do acidentado e cuidador, usando-se a aproximação metodológica do capital humano. A incapacidade causada por acidentes de trânsito tem um alto custo para o sistema de saúde e sociedade mexicana. Baseando-se na perspectiva do sistema de saúde, o custo em pesos mexicanos da incapacidade permanente foi de US\$269.529.480,72, equivalente a US\$1.496,33 por pessoa. Com base na perspectiva da sociedade, estimou-se em US\$3.445,45 no primeiro ano. Em média, estimou-se um custo total de US\$4.941,77 por pessoa, resultando num total de US\$1.119.761.632,53 em 2012. Os resultados deste estudo evidenciam a necessidade de delinear e implementar políticas mais rígidas no México.

Pessoas com Deficiência; Consequências de Acidentes; Acidentes de Transito; Efeitos Psicossociais da Doença 


\section{Colaboradores}

P. G. Sánchez-Vallejo, R. Pérez-Núñez e I. Heredia-Pi concibieron el estudio, analizaron e interpretaron la información. P. G. Sánchez-Vallejo escribió el primer borrador. R. Pérez-Núñez e I. Heredia-Pi revisaron críticamente ese primer borrador y escribieron parte de él. Los tres aprobaron la última versión.

\section{Referencias}

1. World Health Organization. Age-standardized death rates by cause and region, 2004. Global Health Observatory. http://gamapserver.who.int/ gho/interactive_charts/mbd/deaths/chart.html (accedido el 14/Oct/2010).

2. World Health Organization. The top 10 causes of death. Fact sheet № 310. 2008. http://www.who. $\mathrm{int} /$ mediacentre/factsheets/fs310/en/print.html (accedido el 14/Oct/2010)

3. World Health Organization. Part II: global health indicators 2010. http://www.who.int/whosis/ whostat/EN_WHS10_Part2.pdf (accedido el 14/ Oct/2010).

4. World Health Organization. Global burden of disease. Death and DALY estimates for 2004 by cause for WHO Member States, 2009. http://www.who. int/healthinfo/global_burden_disease/estimates_ country/en/index.html (accedido el 08/Feb/2011)

5. Instituto Nacional de Estadística y Geografía. Causas de defunción. Defunciones generales totales por principales causas de mortalidad. 2008. http://www.inegi.org. mx/sistemas/sisept/De fault.aspx?t=mdemo125\&s=est \&c=23589 (accedido el 14/Oct/2010).

6. Bambaren Alatrista C. Características epidemiológicas y económicas de los casos de accidentes de tránsito atendidos en el Hospital Nacional Cayetano Heredia. Rev Méd Hered 2004; 15:30-6.

7. Pérez-Núñez R, Híjar M, Celis A, Hidalgo-Solórzano E. Lesiones causadas por el tránsito: Hora de poner el freno. Cuernavaca: Instituto Nacional de Salud Pública; 2012.

\section{Agradecimientos}

Este trabajo fue parcialmente financiado por CONACYTMéxico a través de una beca para obtener el grado de Maestría en Ciencias de la primera autora (\#230066) Los autores agradecen la revisión y comentarios realizados al protocolo de investigación por Gustavo Rivera Peña, Belkis Aracena, Elisa Hidalgo-Solórzano y Raquel Abrantes, y a Martha Híjar por revisar y comentar una versión preliminar de este documento. Agradecemos también los comentarios recibidos por los pares revisores. Los resultados, opiniones y conclusiones son responsabilidad exclusiva de los autores.
8. Organización Panamericana de la Salud. Informe sobre el Estado de la Seguridad Vial en la Región de las Américas. Washington DC: Organización Panamericana de la Salud; 2009.

9. Pérez-Núñez R, Ávila-Burgos L, Hijar-Medina M, Pelcastre-Villafuerte B, Celis A, Salinas-Rodríguez A. Economic impact of fatal and non-fatal road traffic injuries in Guadalajara Metropolitan Area and Jalisco, Mexico. Inj Prev 2011; 17:297-303.

10. Ávila-Burgos L, Medina-Solis CE, Pérez-Nuñez R, Híjar-Medina M, Aracena-Genao B, HidalgoSolórzano E, et al. Prevalencia de accidentes de tránsito no fatales en México: resultados de la ENSANUT 2006. Salud Pública Méx 2008; 50 Suppl 1:S38-47.

11. Pérez-Núñez R, Híjar-Medina M, Heredia-Pi I, Jones S, Silveira-Rodrigues EM. Economic impact of fatal and nonfatal road traffic injuries in Belize in 2007. Rev Panam Salud Pública 2010; 28:326-36.

12. World Health Organization. World report on disability 2011. Geneva: World Health Organization; 2011.

13. Instituto Nacional de Estadística y Geografía. Las personas con discapacidad en México: una visión censal. México DF: Instituto Nacional de Estadística y Geografía; 2004.

14. Valdespino JL, Olaiz G, López-Barajas MP, Mendoza L, Palma O, Velázquez O, et al. Encuesta Nacional de Salud 2000. Tomo I. Vivienda, población y utilización de servicios de salud. Cuernavaca: Instituto Nacional de Salud Pública; 2003. 
15. States JD, Viano DC. Injury impairment and disability scales to assess the permanent consequences of trauma. Accid Anal Prev 1990; 22: 151-60.

16. World Health Organization. International classification of functioning, disability and health (ICF) [CD-ROM]. Geneva: World Health Organization; 2001.

17. Hernández-Jaramillo J, Hernández-Umana ID. Una aproximación a los costos indirectos de la discapacidad en Colombia. Rev Salud Pública (Bogotá) 2005; 7:130-44.

18. Hijar M, Arredondo A, Carrillo C, Solorzano L. Road traffic injuries in an urban area in Mexico. An epidemiological and cost analysis. Accid Anal Prev 2004; 36:37-42.

19. Mayou R, Bryant B. Consequences of road traffic accidents for different types of road user. Injury 2003; 34:197-202.

20. Gutiérrez JP, Rivera-Dommarco J, Shamah-Levy T, Villalpando-Hernández S, Franco A, Cuevas-Nasu L, et al. Encuesta Nacional de Salud y Nutrición 2012. Resultados nacionales. 2a Ed. Cuernavaca: Instituto Nacional de Salud Pública; 2013.

21) Romero-Martínez M, Shamah-Levy T, Franco A, Villalpando S, Cuevas-Nasa L, Gutiérrez JP, et al. Encuesta Nacional de Salud y Nutrición 2012: diseño y cobertura. Salud Pública Méx 2013; 55 Suppl 2:S332-40.

22. Drummond MF. Methods for the economic evaluation of health care programmes. 2nd Ed. New York: Oxford University Press; 1997.

23. Drummond F, McGuire A. Economic evaluation in health care: merging theory with practice. New York: Oxford University Press; 2001.

24. Instituto Nacional de Estadística y Geografía. Encuesta Nacional de Ingresos y Gastos en los Hogares. 2010. http://www.inegi.org.mx/est/conte nidos/Proyectos/Encuestas/Hogares/regulares/ Enigh/Enigh2010/tradicional/default.aspx (accedido el 03/Jul/2013).

25. Diario Oficial de la Federación. Índice nacional de precios al consumidor. http://dof.gob.mx/nota detalle.php?codigo $=5235380 \&$ fecha $=27 / 02 / 2012$ (accedido el 30/Jul/2013).

26. Hospital General Dr. Manuel Gea González. Tabulador de cuotas de recuperación, 2012. http:// www.hospitalgea.salud.gob.mx/descargas/Tabcuotas-recup-2012.pdf (accedido el 28/May/2013).

27. World Health Organization. WHO-CHOICE Unit costs estimates for service delivery. 2008. http:// www.who.int/choice/country/country_specific/ en/index.html (accedido el 28/May/2012).

28. Diario Oficial de la Federación. Costos unitarios por nivel de atención médica para 2012 del Instituto Mexicano del Seguro Social. https://www. google.com.mx/?gws_rd=ssl\#q=Diario+Oficial+d e+la+Federaci\%C3\%B3n.+Costos+unitarios+por+ nivel+de+atenci\%C3\%B3n+m\%C3\%A9dica+para+ 2012+del+Instituto+Mexicano+del+Seguro+Social (accedido el 30/Jul/2013).
29. Ávila-Burgos L, Ventura-Alfaro C, Barroso-Quiab A, Aracena-Genao B, Cahuana-Hurtado L, ServánMori E, et al. Las lesiones por causa externa en México. Lecciones aprendidas y desafíos para el Sistema Nacional de Salud Mexicano. http://www. insp.mx/images/stories/Produccion/pdf/101203_ cenapra.pdf (accedido em 30/Jul/2013).

30. Instituto Nacional de Salud Pública. Encuesta Nacional sobre Percepción de Discapacidad en la Población Mexicana 2010. Informe final de resultados. http://www.insp.mx/images/stories/Produccion/pdf/131011_ENPDis.pdf (accedido el 07/ Feb/2014).

31. Consejo Nacional de Población. Proyecciones de la población nacional 2010-2050. http://www. conapo.gob.mx/es/CONAPO/Proyecciones (accedido el 30/Jul/2013).

32. Nigenda G, López-Ortega M, Matarazzo C, JuárezRamfrez C. La atención de los enfermos y discapacitados en el hogar. Retos para el sistema de salud mexicano. Salud Pública Méx 2007; 49:286-94.

33. Secretaría de Administración Tributaria. Cuadro histórico de los salarios mínimos (1982-2012). http://www.sat.gob.mx/informacion_fiscal/tablas_indicadores/Paginas/salarios_minimos.aspx (accedido el 30/Jul/2013).

34. Secretaría de Desarrollo Social. Montos mensuales correspondientes al primer semestre de 2013. http://www.oportunidades.gob.mx/Portal/wb/ Web/oportunidades_montos (accedido el 30/ $\mathrm{Jul} / 2013)$.

35. Albert T, Cloutier E. The economic burden of unintentional injury in Alberta. Edmonton: Alberta Centre for Injury Control and Research; 2002

36. Bastida JL, Aguilar PS, González BD. The economic costs of traffic accidents in Spain. J Trauma 2004; 56:883-9.

37. Lin T, Li N, Du W, Song X, Zheng X. Road traffic disability in China: prevalence and socio-demographic disparities. J Public Health (Oxf) 2013; 35:541-7.

38. Ditsuwan V, Veerman LJ, Barendregt JJ, Bertram M, Vos T. The national burden of road traffic injuries in Thailand. Popul Health Metr 2011; 9:2.

39. Juillard C, Labinjo M, Kobusingye O, Hyder AA. Socioeconomic impact of road traffic injuries in West Africa: exploratory data from Nigeria. Inj Prev 2010; 16:389-92.

40. World Health Organization. Global status report on road safety 2013: supporting a decade of action. Geneva: World Health Organization; 2013.

41. Pinet L. Atención prehospitalaria de urgencias en el Distrito Federal: las oportunidades del sistema de salud. Salud Pública Méx 2005; 47:64-71.

42. Mock C, Arreola-Risa C, Quansah R. Strengthening care for injured persons in less developed countries: a case study of Ghana and Mexico. Inj Control Saf Promot 2003; 10:45-51.

Recibido el 10/Feb/2014

Versión final presentado el 07/Ago/2014

Aprobado el 15/Nov/2014 